SHORT REPORT

\title{
Utility of an ultrafast magnetic resonance imaging protocol in recent and semi-recent strokes
}

\author{
J M U-King-Im, R A Trivedi, M J Graves, K Harkness, H Eales, I Joubert, B Koo, N Antoun, \\ E A Warburton, J H Gillard, J-C Baron
}

See Editorial Commentary, p 905

J Neurol Neurosurg Psychiatry 2005;76:1002-1005. doi: 10.1136/jnnp.2004.046201

\begin{abstract}
Objective: To evaluate the technical feasibility of an integrated ultrafast head magnetic resonance (MR) protocol using a sensitivity encoding (SENSE) technique for depicting parenchymal ischaemia and vascular compromise in patients with suspected recent stroke.

Methods: 23 patients were evaluated with the ultrafast MR protocol using $\mathrm{T} 2, \mathrm{Tl}$, fluid attenuated inversion recovery (FLAIR), 3D time of flight magnetic resonance angiography (MRA), and diffusion weighted imaging (DWI) sequences. These were compared with routine conventional MR sequences.

Results: One patient could not tolerate conventional imaging, although imaging using the three minute head SENSE protocol was diagnostic. Both conventional and ultrafast protocols were of similar diagnostic yield in the remaining patients. There were no significant differences in clinical diagnostic quality for the $\mathrm{T} 1, \mathrm{~T} 2$, FLAIR, and DWI sequences. One MRA examination was of better quality when SENSE was used, owing to reduced motion artefacts and shorter imaging time.

Conclusions: It is possible to undertake a comprehensive MR examination in stroke patients in approximately three to five minutes. Ultrafast imaging may become a useful triage tool before thrombolytic therapy. It may be of particular benefit in patients unable to tolerate longer sequences. Further work is necessary to confirm these findings in hyperacute stroke.
\end{abstract}

C omputed tomography (CT) remains the method of choice in the assessment of acute strokes. ${ }^{1}$ It is practical, quick, widely available, easily performed in ill patients, and moreover-as used in the major trials of thrombolysis for acute strokes-it can robustly exclude haemorrhage in the early stages. ${ }^{2}$ Currently, however, there is growing interest in the use of magnetic resonance imaging (MRI) for acute strokes because of the greater sensitivity and interobserver agreement of diffusion weighted imaging (DWI) for detecting early ischaemic signs compared with CT. ${ }^{3}$

Developments in MRI over the past decade now allow the identification of major vascular occlusions and the delineation of tissue at risk of infarction in patients presenting with acute stroke. ${ }^{4}$ MRI can identify parenchymal abnormalities (using $\mathrm{T} 1, \mathrm{~T} 2$, and fluid attenuated inversion recovery (FLAIR) sequences), vascular compromise (magnetic resonance angiography (MRA)), and any DWI/perfusion weighted (PWI) mismatch, providing valuable additional information which helps to refine patient selection and thus to identify subgroups which may benefit most from thrombolysis. ${ }^{5}$

The practicalities of MR imaging of stroke patients acutely, however, remain challenging even for major academic centres. Although there are various explanations for this, one specific problem is the length of time it takes to acquire conventional MR data, which may be especially problematic in restless and uncooperative patients. At 1.5 Tesla, imaging times of 15 to 20 minutes are quite normal. Adding an acute stroke study can be extremely disruptive to the running of MR schedules and so there may be some reticence in encouraging such studies.

Fast MR protocols using echo-planar DWI/PWI have previously been shown to be feasible and practical in the setting of acute stroke. ${ }^{6}$ More recently, advances in coil design and processing using parallel imaging techniques such as SMASH and SENSE $^{7}$ have the potential to reduce imaging times dramatically, with only a small reduction in signal to noise ratio (SNR). Such techniques allow the acquisition of T1, T2, FLAIR, DWI, and 3D time of flight MRA in approximately three to five minutes.

The purpose of this technical feasibility study was to evaluate the diagnostic yield and quality of images obtained with an ultrafast MRI protocol based on SENSE, compared with our conventional MR protocol, in the setting of suspected recent or semi-recent strokes.

\section{METHODS}

Twenty three patients ( 15 female, eight male; mean age 76 years, range 39 to 90 ) with a clinical diagnosis of suspected recent or semi-recent strokes were imaged using the ultrafast MR protocol as well as our conventional protocol, employing an eight channel phased array brain coil on a 1.5T MR system (Signa Echospeed+ EXCITE, GE Medical Systems, Milwaukee, Wisconsin, USA). The sequences were obtained as part of routine clinical evaluation of MR sequences where all clinical patients underwent both conventional and faster sequences in a single clinical examination. The local research and ethics committee approved release of these clinical data for this study.

Examinations included a T1, T2, FLAIR, and diffusion weighted sequences in addition to a three dimensional time of flight MRA. For clarity, the parameters have been outlined in table 1. In order to minimise schedule disruption patients were all imaged in the next available clinical session. The mean time from stroke onset to imaging was 165.6 hours, with a standard deviation and standard error of 125.7 and 26.87 hours, respectively. All patients waking with stroke symptoms were presumed to have had their stroke when they were definitely well before going to sleep.

In the ultrafast protocol, all images were acquired using a proprietary implementation of SENSE (ASSET, GE Medical Systems) with a scan reduction factor of 2 in the in-plane

Abbreviations: DWI, diffusion weighted imaging; FLAIR, fluid attenuated inversion recovery; MRA, magnetic resonance angiography; $\mathrm{PWI}$, perfusion weighted imaging; SENSE, sensitivity encoding; SMASH, simultaneous acquisition of spatial harmonics; SNR, signal to noise ratio 


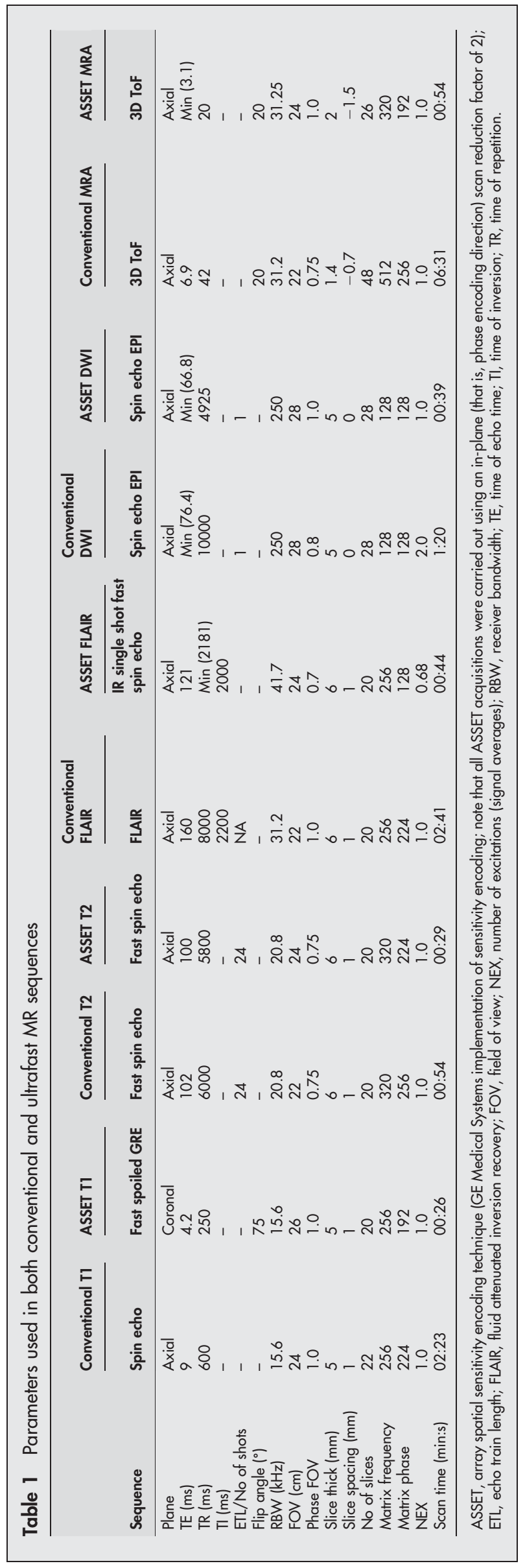

phase encoding direction. In some sequences we allowed a slight increase in the field of view to ensure that there was no image aliasing before application of the ASSET technique. Before the acquisition of the ASSET sequences a single six second ASSET calibration scan in the sagittal plane was acquired. The post-processing time for the ultrafast MRA examination was approximately seven minutes. During this time, other sequences were filmed and the patient was removed from the room and returned to their inpatient bed.

An attending neuroradiologist and a stroke neurologist, blinded to the clinical information, independently evaluated the ultrafast imaging protocol and the conventional images for diagnostic yield with respect to parenchymal abnormalities, major vascular occlusions, and any suggestions of haemorrhage. Image quality was graded by the attending neuroradiologist only, on a four point scale (grade 1 , excellent; grade 2, more than adequate for diagnosis; grade 3, adequate for diagnosis; grade 4, inadequate for diagnosis), based on various criteria which included spatial resolution, SNR, conspicuity of lesions, and artefacts (movement and ghosting).

Statistical analysis was carried out with SPSS for Windows (version 10.1; SPSS Inc, Chicago, Illinois, USA), using Fischer's exact test and with significance defined as $p \leqslant 0.05$.

\section{RESULTS}

Seventeen patients had a final diagnosis of a recent middle cerebral artery stroke, two had a lacunar stroke, three had a posterior circulation stroke, and one had negative MR imaging with a final diagnosis of a transient ischaemic attack. One patient was unable to complete the standard protocol owing to claustrophobia; the ultrafast protocol was subsequently undertaken and produced a diagnostic study, showing a right basal ganglia DWI abnormality with patent major basal cerebral arteries.

Conventional MRA demonstrated occlusion or reduced MCA flow in seven cases; the ultrafast MRA was positive in six cases only, as judged by both readers. In one case, vascular narrowing was identified on conventional MRA by both readers but only by the attending neuroradiologist on ultrafast MRA. Subtle vascular narrowing was confirmed to be present on a retrospective consensus review on both MR studies. One ultrafast MRA protocol was of better quality than the conventional sequence, probably reflecting the shorter imaging time and decrease in motion artefacts (fig 1). Two ultrafast MRAs (grade 3) were considered to be of lesser quality than the conventional MRA (grade 2) because of reduced spatial resolution, reflecting the reduced acquisition time, although this did not affect diagnostic value. An incidental middle cerebral artery aneurysm was demonstrated with both protocols. Seventy per cent of conventional MRAs scored an image quality of grade 2 or better, compared with $65 \%$ of the ultrafast MRAs, but this difference was not statistically significant $(p=1.00)$.

Both independent readers were in $100 \%$ agreement regarding the presence or absence of parenchymal abnormalities on DWI. Moreover, the DWI study was in 100\% agreement using both protocols. In terms of image quality, $96 \%$ of the ultrafast DWI studies scored grade 2 or better, compared with $85 \%$ for the conventional DWI studies but this difference did not reach statistical significance $(p=0.80)$. This was because, although ultrafast DWI sequences had slightly reduced spatial resolution, they also benefited from appreciable reduction in inhomogeneity artefacts at the base of the brain or the regions of the sinuses, owing to the reduced echo train length.

Similarly, all FLAIR, T1, and T2 weighted studies were of similar diagnostic yield for parenchymal abnormalities with respect to both protocols, despite the ultrafast sequences 


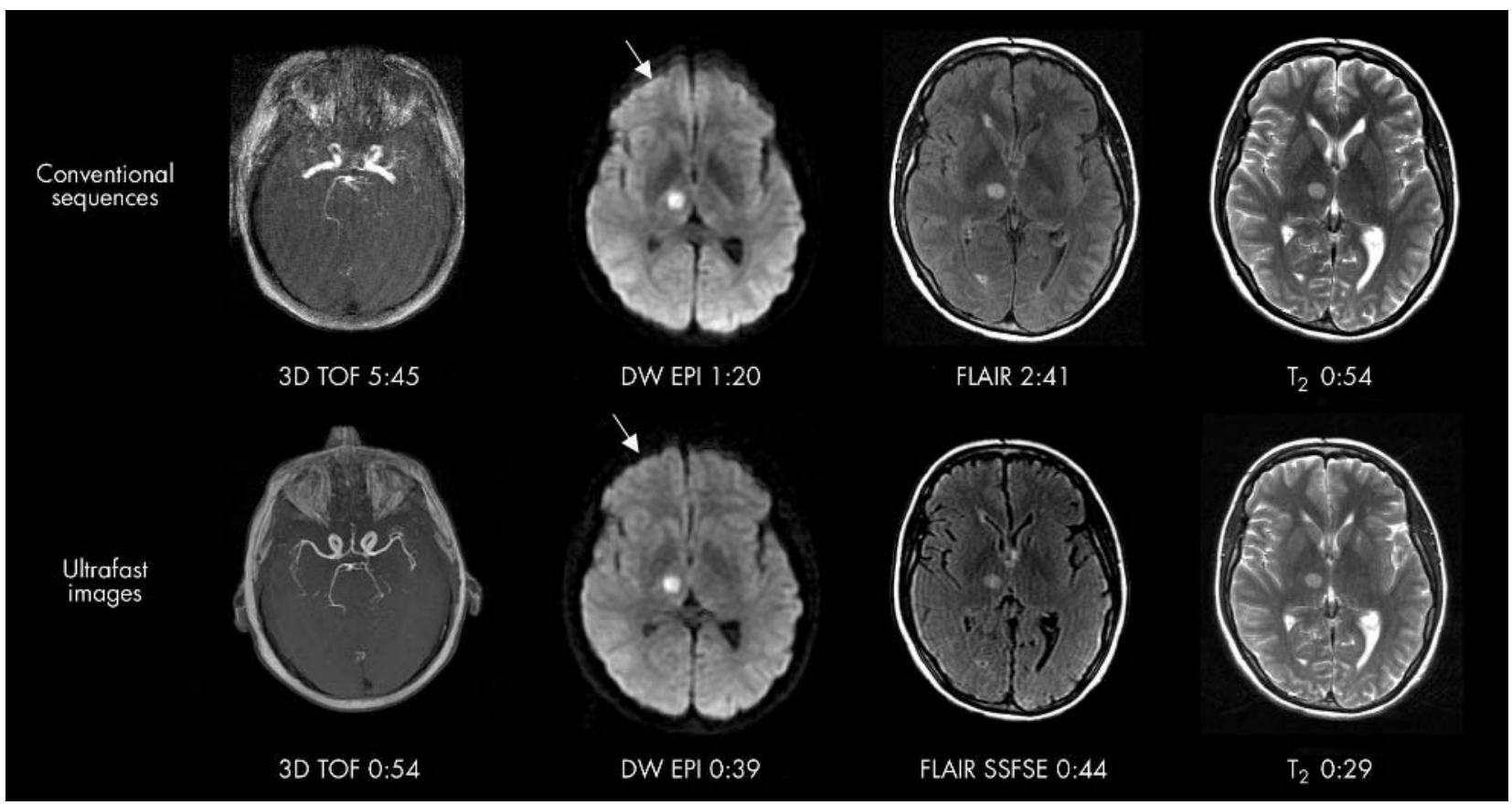

Figure 1 Conventional (top row) and ultrafast imaging acquired with SENSE (bottom row) clearly demonstrates a right thalamic infarct. The three dimensional time of flight MRA acquired using SENSE technology in 54 seconds shows improved definition of the major middle cerebral arteries when compared with the conventional MRA sequence acquired over 6 minutes, 31 seconds. In addition the SENSE diffusion weighted sequence demonstrates slightly reduced distortion over the frontal lobes when compare with conventional sequences (arrows).

suffering from marginally decreased SNR. No statistically significant differences in image quality were, however, observed. Eighty seven per cent of conventional FLAIR images scored an image quality of grade 2 or better, compared with $78 \%$ of the ultrafast studies $(p=0.69) ; 91 \%$ of conventional T2 images scored an image quality of grade 2 or better compared with $78 \%$ of the ultrafast studies $(p=0.41)$; and $83 \%$ of conventional Tl images scored an image quality of grade 2 or better compared with $83 \%$ of the ultrafast studies $(\mathrm{p}=1.00)$.

\section{DISCUSSION}

The recent availability of multichannel phased array brain coils and the development of parallel imaging techniques such as SENSE allow a comprehensive MR stroke imaging protocol to be completed in approximately three to five minutes. We have shown in this study that any reduction in image quality with such a protocol is minimal and does not affect diagnostic yield. The addition of an exogenous perfusion sequence would add an extra 90 seconds as the technique is limited by physiologythat is, by the time required for the contrast agent bolus to transit the brain - and not by the scanner acquisition speed. In addition, there are obvious benefits of including a haemosiderin sensitive gradient echo sequence to use with the Tl and FLAIR sequences to increase the sensitivity of MR for detecting early haemorrhages. ${ }^{10}$ Such echo-planar gradient echo sequences are, however, already ultrafast MR techniques. Thus, in this technical feasibility study we did not evaluate the use of PWI or haemosiderin sensitive sequences, as these are not expected to benefit significantly from parallel imaging techniques. ${ }^{1}$

Recent work has shown that T2, FLAIR, and DWI used with SENSE in patients with acute and subacute stroke (5-42 hours from stroke onset) produces images of equal imaging quality and lesion conspicuity when compared with conventional images. ${ }^{11}$ Our study complements the work of Willinek et al, showing that MRA using SENSE technology can provide evidence of major vascular compromise in a very short acquisition time. ${ }^{11}$ In our MR protocol, the gain in imaging time with sense MRA was partly achieved at the expense of slightly reduced spatial coverage but this did not affect diagnostic yield. We have shown that in two of our cases there were additional benefits from using SENSE: first, we successfully imaged a patient whose claustrophobia precluded her staying in the magnet for the conventional sequences; and second, there was a case where the SENSE MRA was of better quality than the conventionally acquired MRA (fig 1).

In this study we used parallel imaging to reduce the total imaging time to one appropriate for studies where rapid imaging is essential so as not to compromise the rigid temporal time restraints for treatment. Compared with conventional MR imaging, the shorter imaging sequences allow the development of imaging protocols in acute stroke which are less disruptive to scheduling, making MR imaging of acute stroke victims more feasible and potentially allowing better targeted treatment.

A limitation of our study is that our patient population consisted mainly of acute and subacute strokes but did not include hyperacute strokes ( $<6$ hours). It is possible that in patients with hyperacute strokes, who tend to be generally more agitated, the benefits of ultrafast MR protocols may be even more significant, as reduction in motion artefacts is more likely to outweigh any marginal decrease in spatial resolution and SNR. ${ }^{11}$ On the other hand, however, it is possible that in hyperacute strokes, DWI abnormalities as well as radiological signs such as hyperdense cerebral arteries on FLAIR imaging may be more subtle than in the population evaluated in our study. A final limitation is that our study population did not include patients with brain stem infarction.

\section{Conclusions}

Ultrafast MR protocols using SENSE appear very promising but further work in a larger cohort of patients with hyperacute strokes will be necessary to confirm their feasibility, diagnostic accuracy, and clinical utility in a real world practical setting. 


\section{ACKNOWLEDGEMENTS}

We are grateful for the support of the Fund for Addenbrooke's and GE Medical Systems. We thank Claire Sims and Diana Day for their help. RAT and JUKI were supported by the Stroke Association and GlaxoSmithKline.

\section{Authors' affiliations}

J M U-King-Im, R A Trivedi, M J Graves, H Eales, I Joubert, B Koo, N Antoun, J H Gillard, Department of Radiology, Addenbrooke's

Hospital and the University of Cambridge, Cambridge, UK

K Harkness, E A Warburton, J-C Baron, Department of Neurology, Addenbrooke's Hospital and the University of Cambridge

Competing interests: none declared

Correspondence to: Dr Jonathan H Gillard, Department of Radiology, Addenbrooke's Hospital and the University of Cambridge, Cambridge CB2 2QQ, UK; ihg21@cam.ac.uk

Received 23 May 2004

In revised form 7 December 2004

Accepted 8 December 2004

\section{REFERENCES}

1 Wardlaw JM, Farrall AJ. Diagnosis of stroke on neuroimaging. BMJ 2004;328:655-6.

2 Hacke W, Donnan G, Fieschi C, et al. Association of outcome with early stroke treatment: pooled analysis of ATLANTIS, ECASS, and NINDS rt-PA stroke trials. Lancet 2004;363:768-74.

3 Lev MH. CT versus MR for acute stroke imaging: is the "obvious" choice necessarily the correct one? Am J Neuroradiol 2003;24:1930-1.

4 Sorensen AG, Buonanno FS, Gonzalez RG, et al. Hyperacute stroke: evaluation with combined multisection diffusion-weighted and hemodynamically weighted echo-planar MR imaging. Radiology 1996;199:391-401.

5 Albers GW, Clark WM, Madden KP, et al. ATLANTIS trial. Results for patients treated within 3 hours of stroke onset. Stroke 2002;33:493-6.

6 Sunshine JL, Tarr RW, Lanzieri CF, et al. Hyperacute stroke: ultrafast MR imaging to triage patients prior to therapy. Radiology 1999;212:325-32.

7 Sodickson DK, Manning WJ. Simultaneous acquisition of spatial harmonics (SMASH): fast imaging with radiofrequency coil arrays. Magn Reson Med 1997;38:591-603.

8 Pruessmann KP, Weiger M, Scheidegger MB, et al. SENSE: sensitivity encoding for fast MRI. Magn Reson Med 1999;42:952-62.

9 van den Brink JS, Watanabe Y, Kuhl CK, et al. Implications of SENSE MR in routine clinical practice. Eur J Radiol 2003;46:3-27.

10 Fiebach JB, Schellinger PD, Gass A, et al. Stroke magnetic resonance imaging is accurate in hyperacute intracerebral hemorrhage: a multicenter study on the validity of stroke imaging. Stroke 2004;35:502-6.

11 Willinek WA, Gieseke J, von Falkenhausen M, et al. Sensitivity encoding for fast MR imaging of the brain in patients with stroke. Radiology 2003;228:669-75 